\title{
A Statistical DOI Estimation Algorithm for a SiPM-Based Clinical SPECT Insert
}

\author{
Ilenia D’Adda, Student Member, IEEE, Ashley J. Morahan, Marco Carminati, Senior Member, IEEE, \\ Kjell Erlandsson, Michael Ljungberg, Brian F. Hutton, Senior Member, IEEE, \\ and Carlo Fiorini, Senior Member, IEEE
}

\begin{abstract}
A prototype clinical brain SPECT insert has been designed for use in simultaneous SPECT/MRI. The system utilises novel slit-slat collimators which, like pinhole collimators, suffers from parallax errors due to the large incident angle of photons. A statistical algorithm has been developed to determine the depthof-interaction (DOI) with a view to improving image performance. The importance of DOI correction was demonstrated using Monte Carlo simulation. This simulation also indicated that 4 DOI layers $(3 \times 1.5 \mathrm{~mm}+3.5 \mathrm{~mm})$ may be sufficient. The improvement in event localisation was demonstrated on a single detector before implementing the algorithm on the full clinical prototype where some limitations in event localisation in layers close to the readout plane were observed. Nevertheless DOI enabled the rejection of poorly localised events with improved resolution in reconstructed line sources.
\end{abstract}

Index Terms-SPECT, depth-of-interaction, event localisation.

\section{INTRODUCTION}

$\mathbf{W}$ E have previously reported on the development of a compact stationary single photon emission computed tomography (SPECT) system, specifically designed as an insert for acquisition of brain SPECT simultaneously with MRI [1] [2]. The system uses detectors composed of $8 \mathrm{~mm}$ thick CsI scintillators coupled with custom-built silicon photomultiplier (SiPM) readout and consists of 20 detectors (each $10 \mathrm{~cm}$ by $5 \mathrm{~cm}$ ) arranged in a partial ring that fits in the bore of any standard clinical MRI system. A novel multi-slit-slat (MSS) collimator was designed with slits internal to the slat length so as to achieve transaxial minimisation without compromising the slat length that defines axial resolution (see Fig. 1). The INSERT system has undergone preliminary evaluation as a standalone system [3].

A recognised problem that occurs with slit-slat and pinhole collimators is the uncertainty in localising events in the detector due to parallax (Fig. 2). Unlike parallel-hole collimators where incident photons travel (close to) normal to the detector surface, in the case of slit-slat and pinhole

Manuscript received September 13, 2021. This work did not involve human subjects or animals in its research. The first two authors contributed equally to this work.

I. D'Adda, M. Carminati, and C. Fiorini are with Dipartimento di Elettronica, Informazione e Bioingegneria, Politecnico di Milano, Milano, 20133, Italy and with Istituto Nazionale di Fisica Nucleare (INFN), Sezione di Milano, Milano, 20133, Italy (email: marco1.carminati@ polimi.it).

A. J. Morahan, K. Erlandsson and B. F. Hutton are with University College London, London, UK.

M. Ljungberg is with Medical Radiation Physics, Department of Clinical Sciences, Lund University, Lund, Sweden. collimators the incident photons have a range of trajectories that is defined by the opening angle of the aperture. The uncertainty in determining location leads to a loss of resolution in the acquired projections. If the depth-of-interaction (DOI) is known then the correct trajectory can be identified, addressing the problem. Methods for determining DOI have been developed mainly for application in positron emission tomography (PET) to address the uncertainty in the line-ofresponse for coincidence events that can occur toward the edge of the field-of-view, especially on small diameter PET systems [4] and with monolithic scintillators [5], [6]. In this context, machine learning has been primarily leveraged [7], [8], in particular gradient trees [9]. However, there has been much lower demand for DOI determination for SPECT where spatial resolution is already compromised by the physical collimation. There is also little known regarding the effect of parallax errors on SPECT reconstruction, especially when complex acquisition geometry is involved.

In this paper we propose an approach for DOI determination specifically for SPECT. Variegated solutions have been proposed to address the parallax error in SPECT with pinhole collimators, including laser machining of the scintillator [10] as well as curved scintillator [11]. Despite effective, these approaches show limited potential for scale-up, given the cost and complexity of the fabrication. Instead, we propose a statistical method to be applied to standard scintillators based of a novel mapping parameter as DOI proxy, improved with respect to the N/I ratio proposed in the past [12]. The paper is organised as follows. We first present the results of Monte Carlo (MC) simulations where the effects of parallax errors and DOI correction are estimated for data acquired on the INSERT system. The MC data are also used to determine the sufficient number of discrete DOI layers that are required.
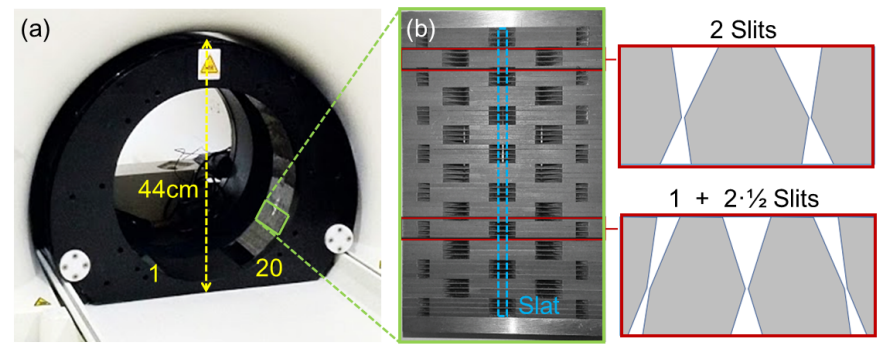

Fig. 1. Clinical SPECT INSERT (a) inside a MRI scanner, (b) collimator design. 
TABLE I

MEAN FWHM OF LINE SOURCES RECONSTRUCTED FROM VARIOUS DOI LAYER CONFIGURATIONS.

\begin{tabular}{l|c|c|c}
\hline DOI Config. & Radius [mm] & Long Axis [mm] & Short Axis [mm] \\
\hline $1(1 \times 8 \mathrm{~mm})$ & 30 & $6.97 \pm 0.25$ & $5.97 \pm 0.13$ \\
& 60 & $7.18 \pm 0.42$ & $5.73 \pm 0.21$ \\
& 90 & $8.09 \pm 0.60$ & $5.12 \pm 0.78$ \\
\hline 4 & 30 & $6.78 \pm 0.25$ & $5.86 \pm 0.12$ \\
$(3 \times 1.5+3.5 \mathrm{~mm})$ & 60 & $6.27 \pm 0.45$ & $5.40 \pm 0.14$ \\
& 90 & $5.89 \pm 0.67$ & $4.42 \pm 0.22$ \\
\hline $8(8 \times 1 \mathrm{~mm})$ & 30 & $6.79 \pm 0.25$ & $5.87 \pm 0.12$ \\
& 60 & $6.23 \pm 0.46$ & $5.39 \pm 0.14$ \\
& 90 & $5.80 \pm 0.67$ & $4.40 \pm 0.20$ \\
\hline
\end{tabular}

A method for determining DOI using maximum likelihood estimation is described, including a practical calibration procedure that can be implemented in the field, with fully assembled scanner. The effectiveness of the algorithm is evaluated initially for a highly collimated source on a single detector. Finally the improvement in spatial resolution is assessed when DOI correction is implemented on the actual INSERT system.

\section{Monte Carlo Simulations}

The INSERT system was modelled within the MC simulation software SIMIND [13], by defining the detector configuration and unique MSS collimator geometry. To determine the effects of DOI we reconstructed a set of simulated line-source data with known DOI information. The parallax error present due to oblique photons can be corrected with the knowledge of each event DOI. We set out to determine the effects of DOI correction in image reconstruction and establish the ideal choice of DOI layers.

It was assumed that the DOI algorithm can position events into discrete DOI layers. The simulation output was therefore sorted into several DOI layer configurations within the $8 \mathrm{~mm}$ crystal. The data were first divided into $160.5-\mathrm{mm}$ thick layers, where each layer is sufficiently thin to minimize uncertainty within the layer. These data were summed to illustrate the effect of the parallax errors. Additionally the crystal was divided into $8 \times 1 \mathrm{~mm}$ layers and a 4 layer configuration $(3 \mathrm{x}$ $1.5+3.5 \mathrm{~mm}$ ) that recognises the reduced events in deeper layers.

The different DOI configurations were evaluated with simulations of line sources $(10 \mathrm{~cm}$ long and $0.01 \mathrm{~mm}$ thick). A set of ${ }^{99 m} \mathrm{Tc}$ line sources were simulated, located radially at 30, 60 and $90 \mathrm{~mm}$ from the centre of the field-of-view in 8 different angular positions. $80 \mathrm{M}$ events were generated from

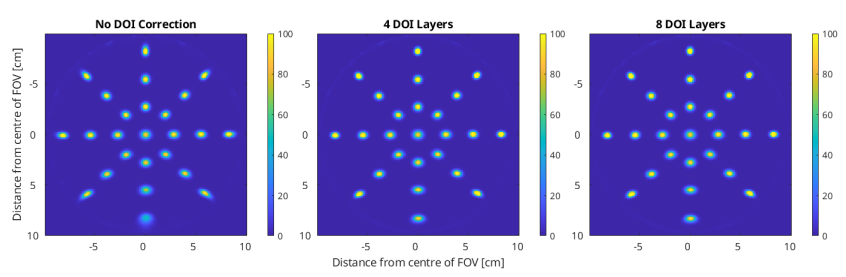

Fig. 2. Capillary images reconstructed from SIMIND data, without DOI (left), using 8 DOI layers (right) and 4 DOI layers (middle). Improvement is quantified in Tab. 1.
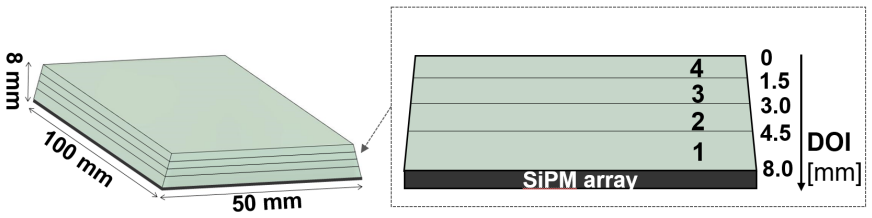

Fig. 3. CsI(Tl) crystal $(50 \mathrm{~mm} \times 100 \mathrm{~mm} \times 8 \mathrm{~mm})$ segmentation of the crystal into DOI layers of respectively $1.5 \mathrm{~mm}$ (layers $2,3,4$ ) and $3.5 \mathrm{~mm}$ (layer 1) thickness.

each capillary. The line spread function (LSF) was plotted at each layer and the parallax error was observed through a shift in peak position for deeper layers. The line source data were corrected for parallax and image reconstruction was carried out using 20 iterations of ML-EM. Short and long axis resolution was estimated for each line source with results presented in Table I and Fig. 2. Improvement in resolution is most apparent for the line sources positioned at $90 \mathrm{~mm}$ radius, including sources positioned in the region where there is lower sampling due the partial ring geometry (Fig. 1). When comparing the different layer configurations the measurement and correction of parallax error was sufficient when few layers were used as illustrated in Table I. The DOI correction was particularly effective in reducing the long axis (radial) resolution for sources positioned at $90 \mathrm{~mm}$ radius. Identification of 4 DOI layers was implemented in the physical system as a reasonable trade-off.

\section{DOI ESTIMATION ALGORITHM}

\section{A. Algorithm Overview}

The proposed procedure for DOI event reconstruction answers the need for a fast calibration procedure, which is implementable with a simple set-up and is viable in clinical operations. This requires that all the modules of the scanner can be calibrated with a single operation without any dedicated pencil-beam scan or additional collimator. The core of the algorithm relies on a statistical method, specifically Maximum Likelihood (ML), to estimate the depth at which events are absorbed. Based on the simulation results, the CsI(Tl) crystal thickness of $8 \mathrm{~mm}$ is segmented into four DOI layers: three of

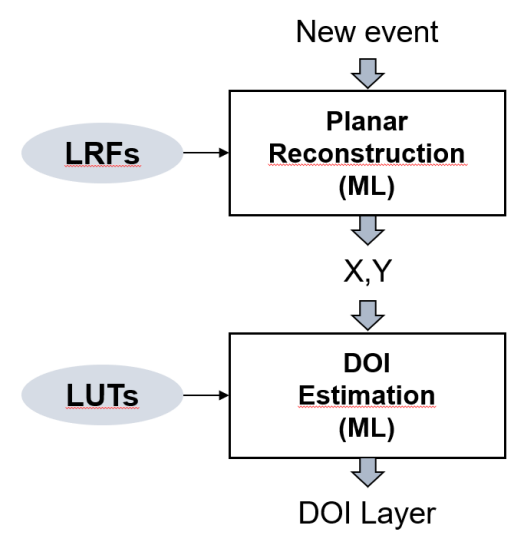

Fig. 4. Block diagram of the ML-based event reconstruction algorithm, which first estimates the $x$ and $y$ coordinates and then classify the event into one DOI layer. 
them have an equal thickness $(1.5 \mathrm{~mm})$ and one layer is 3.5 $\mathrm{mm}$ thick (Fig. 3). Furthermore, a more refined DOI determination is difficult for deeper layers as the light distribution, being more peaked, illuminates only one (or few) SiPM(s) below the scintillation position.

The ML-based position reconstruction method requires a calibration procedure to estimate the forward model describing the expected response of the camera. Traditionally, this estimation was carried out through simulations [14] or complex scanning setups [15], which can be expensive and time consuming. For INSERT detection modules, the forward model used to determine the $x, y$ coordinates of absorbed gamma rays assumes 2D Gaussian Light Response Functions (LRFs) [16]. The adaptive estimation of LRFs only requires a simple flood-field irradiation (FFI) [17], consequently, this approach is preferred since a one-time measurement is enough to calibrate all the 20 modules of the scanner. The extension to the third coordinate (i.e. DOI) is implemented by applying a second stage of processing to the $72 \mathrm{SiPM}$ signals acquired for each detected event. This second stage takes as input the reconstructed $x$ and $y$ coordinates, then, assigns the event to the DOI layer for which a second likelihood function is maximized. The position estimation chain is depicted in Fig. 4.

For DOI reconstruction, the expected response of the detection modules is encoded in a set of Look-Up Tables (LUTs). As the signal acquired by $j$-th channel is assumed to be welldescribed by a normal distribution [18], the LUTs is obtained as:

$$
\operatorname{LUTs}(x, y)=\left\{\left\{\mu_{j}^{(k)}(x, y), \sigma_{j}^{(k)}(x, y)\right\}_{j=1 \ldots 72}\right\}_{k=1 \ldots 4}
$$

where $\mu_{j}^{(k)}(x, y)$ is the mean signal acquired by the $j$-th channel for the $k$-th DOI layer, which is a function of $x$ and $y$ coordinates, and $\sigma_{j}^{(k)}(x, y)$ its variance. Therefore, for the $i$-th gamma event, the log-likelihood of belonging to the $k$-th DOI range can be expressed as:

$\log L\left(\operatorname{layer}_{k} \mid\left\{s_{i j}\right\}_{j=1 \ldots 72}\right)=-\sum_{j=1}^{72}\left\{\frac{\left(s_{i j}-\mu_{j}^{(k)}\right)^{2}}{2 \cdot\left(\sigma_{j}^{(k)}\right)^{2}}+\ln \left(\sqrt{2 \pi} \cdot \sigma_{j}^{(k)}\right)\right\}$

where $s_{i j}$ is the signal acquired by the $j$-th channel for the $i$-th event. The maximization of the logarithmic likelihood (rather than likelihood) is generally more convenient, as it works with sums rather than products. LUTs are generated through a dedicated calibration procedure which again relies only on flood field irradiation, so they are obtained from the FFI dataset already used for LRF computation. This acquisition is performed only one time before actual measurements if operating conditions remain stable throughout the whole acquisition session.

\section{B. Choice of the Initialization Signal}

The starting point for LUT generation relies on an initialization signal with a good correlation and monotonic dependency with the DOI. This aspect, and the entire calibration procedure was studied and optimized by means of the ANTS2 simulation package [19]. The Clinical INSERT detector unit

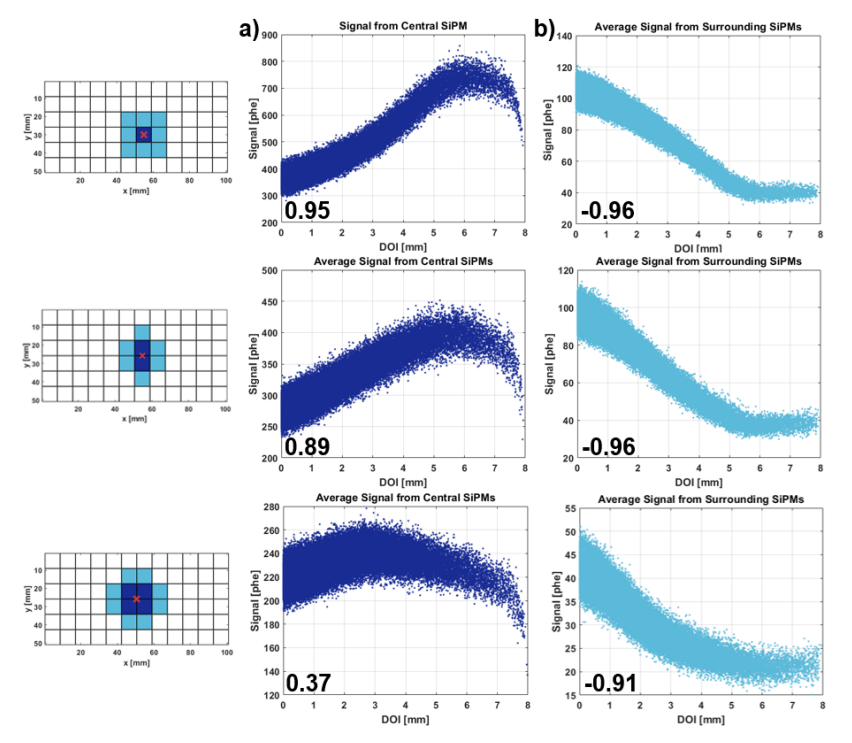

Fig. 5. Simulation data obtained for a spot (red cross) positioned in the center of a SiPM (top row), on the edge (middle) and on the corner (bottom row). The graphs on the right show the trend of the selected initialization signals when varying the DOI (their correlation is reported in the bottom left corner of each plot). Two types of initialization signals are considered: (a) the signal acquired by the channel(s) detecting on average the highest intensity of light (blue) and (b) the average of the signals acquired by the ring of SiPMs around the spot position (cyan). Both are expressed in photo-electrons. For a critical spot position in the corner, (a) has correlation (0.37) while (b) is always strong (higher than 0.9) and thus is selected.

was modelled taking into account both scintillation and SiPMs properties. A point-like mono-energetic $(140 \mathrm{keV})$ collimated gamma source was positioned at determined $x$ and $y$ coordinates. The resulting light distribution footprint on the SiPM array was collected and investigated to identify the optimum initialization signal. This analysis allowed separation of the photo-detector signal variations related to the DOI, from the variations related to $x, y$ coordinates. In particular, three different types of spot positions were analyzed: aligned to the SiPM centers, their edges and the corners. A common strategy implemented by others is to extract the DOI information starting from the intensity of the signal acquired by the channel detecting the majority of light photons, normalized by the sum of the signals collected by the array (i.e. the energy) [20] [21] [22]. As the correlation of this indicator with DOI for our configuration ( $8 \mathrm{~mm}$ crystal thickness, $8.2 \mathrm{~mm} \mathrm{SiPM}$ side) was low for the most critical spot position, i.e. on SiPM corners, we have proposed an alternative novel initialization signal, referred to as $\mu_{\text {ring }}$, which shows a good correlation and a monotonic dependency with the DOI for all three types of spot position (Fig. 5). The selected signal is computed as the average of the signals acquired by the channels surrounding the spot position.

\section{Calibration Procedure}

The calibration procedure is divided into three main steps:

1) SUBDIVISION of events into spots: LRFs, estimated from the calibration measurement (FFI), are used to reconstruct the $x$ and $y$ coordinates of the gamma rays interacting in the scintillator and divide them into 253 virtual spots 4 


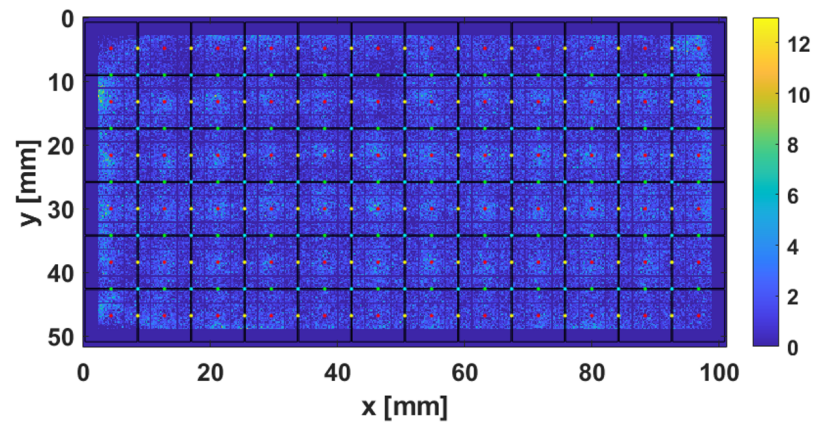

Fig. 6. Flood field irradiation (FFI) acquired for the DOI estimation algorithm training. Events are divided into 253 regions on the basis of their reconstructed $x, y$ coordinates.

mm wide in both directions (Fig. 6). An energy filter is applied to select only photopeak events. The initial choice of the spot size $(1 \mathrm{~mm})$ was increased to gather enough data for every spot while minimizing the time required for the calibration. Then, depending on the position of each virtual spot, the corresponding ring of SiPMs required for the is identified. Partial rings are selected for crystal borders (Fig. 7).

2) GENERATION of discrete LUTs: in this step single spots are processed independently. For every event, $\mu_{\text {ring }}$ is obtained as the mean intensity of the channels surrounding the spot position. Then, the probability for an interaction to happen within a given range in the crystal thickness can be computed from the Lambert-Beer law. A preliminary grouping of events is performed computing the quantiles of the distribution of $\mu_{\text {ring }}$ in the four layers (Fig. 8).

After computing the first set of LUTs values, the initial subdivision is updated through the ML-based approach (described in section III-A) to obtain the final DOI for each event $\left(\mu_{j}^{(k)}(\hat{x}, \hat{y}), \sigma_{j}^{(k)}(\hat{x}, \hat{y})\right.$, where $\hat{x}$ and $\hat{y}$ identify the location of the current spot). Fig. 10 shows the final percentages of correct classification for each DOI layer obtained from simulated data of two different types of spot position.

3) INTERPOLATION: in order to obtain the LUT values as a function of $x, y$ coordinates and describe also intermediate
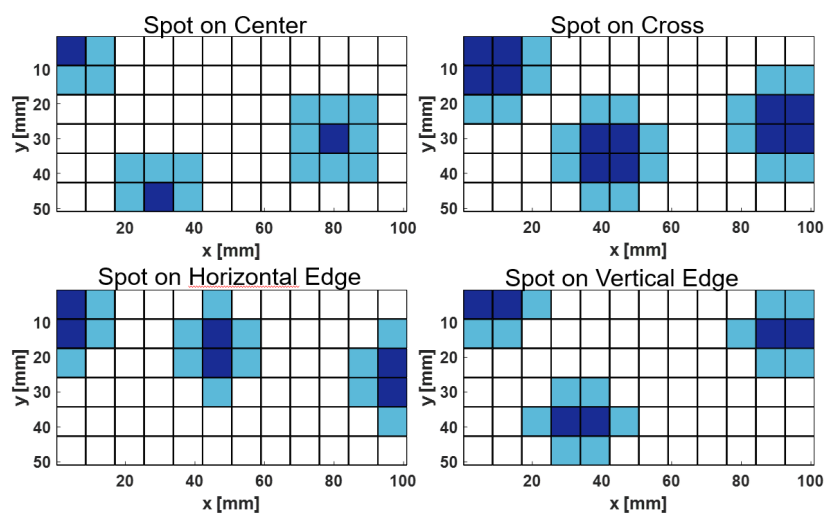

Fig. 7. Adaptive selection of the ring of SiPMs depending on the spot location. Partial rings are used for events at the edges of the FOV.
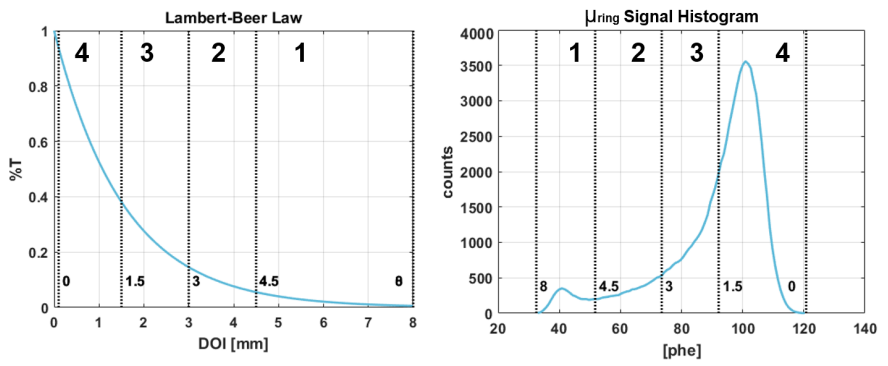

Fig. 8. (left) Theoretical Lambert-Beer law. (right) Distribution of $\mu_{\text {ring }}$. The vertical lines identifies the quantiles of the distribution splitting the events into the four DOI classes.
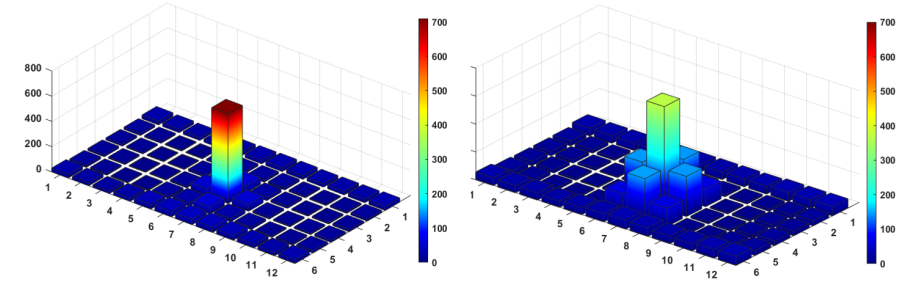

Fig. 9. Simulated data representing the signal acquired by the photodetector array (expressed in photoelectrons) for an event absorbed at high (left) and low (right) DOI.

positions between the spots, the discrete values are interpolated with a 2D fitting method, in particular a LOESS smoothing function based on local regression. It offers better fitting than Gaussian at the edges.

\section{Single Module Experimental Validation}

The algorithm was initially tested on a single clinical detection module. For the experimental validation, the detector was firstly irradiated uniformly to perform the calibration described in section III-C. Then, a ${ }^{57}$ Co source (122 keV photopeak) was collimated by a stack of four single-hole lead collimators (1 $\mathrm{mm}$ diameter hole) placed in a custom 3D-printed holder tilted with respect to surface of the scintillator. In this way gamma rays incident on the camera surface are only the ones tilted by $45^{\circ}$ degrees. The collimated beam is aligned along the $x$-axis thus producing a correlation of the reconstructed $x$ coordinates with the DOI positions (Fig. 11.a).

The planar reconstruction of the tilted irradiation is shown in Fig. 11.b. The elongated right side of the spot has a lower density of counts, as these events are absorbed close to the photodetector array given the oblique geometry. All events interacting at $x>0$ were classified in the four DOI layers and the result is presented in Fig. 11.c. As expected, the peak of each layer population is shifted towards higher $x$ ' values moving from the upper layer to the lower one. As the degree of source collimation decreases with the distance, layer 1 is affected by the highest peak position error. Moreover, when rays are tilted by 45 degrees, the amount of gamma events reaching $x^{\prime}=8 \mathrm{~mm}$ is almost negligible. The correction of the parallax error (in this simplified geometry) is made by applying to each event a backward shift along $x$ ' proportional to the depth of the layer in which the event has been classified. After correcting the elongation of the reconstructed trajectory, 

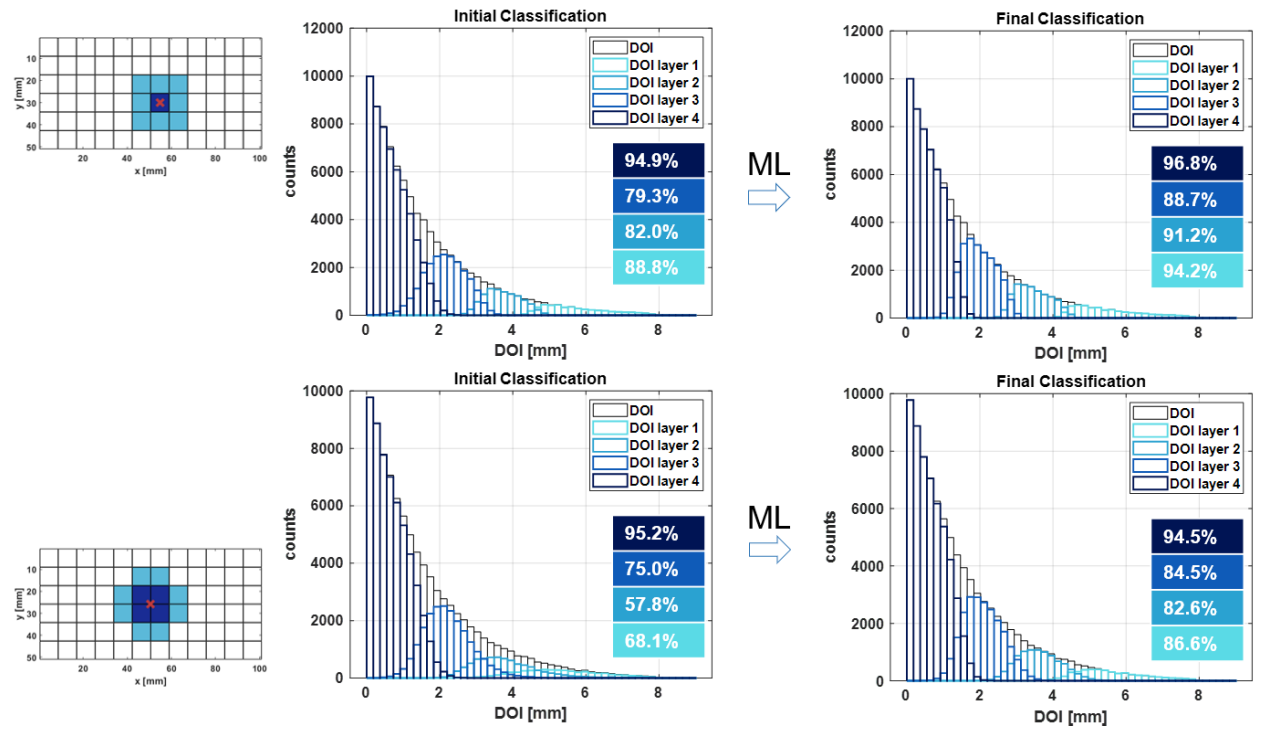

Fig. 10. Percentages of correct classification for each DOI layer estimated from simulated data for an irradiation spot positioned over the center (top) and over the corner (bottom) of a SiPM. The improvement after the Maximum Likelihood-based classification can be clearly seen.

the ratio between the major and minor axes of the ellipse circumscribing the projected spot changes from 2.3 to 1.4 .

\section{EXPERIMENTAL VALidation With Full SCANNER}

The DOI categorization algorithm was applied to data acquired from all 20 modules in the INSERT scanner to assess the effectiveness of DOI correction. Initially the intrinsic uniformity was assessed with a line source placed at the centre of the field of view (FOV), with collimator removed (fig. 12). An evaluation of the individual DOI layers demonstrated that, for several of the detectors, there was poor uniformity for deeper layers, presumably due to a limitation in the event positioning close to the detector. Uniformity and linearity calibration was invalid for deeper DOI layers. But the top two layers, which contain most events and have less parallax error, had more acceptable uniformity. Therefore we chose to use the DOI to simply identify the layers where positioning appeared to be effective. This results in some loss of counts $(33.7 \%)$, but has the potential to improve image quality. An approximation of the uniformity and linearity calibration data was created with the top two layers and used to correct the data in each layer.

A study was performed with a series of line source acquisitions with sources located at a radius of 25, 50, 75 and 100 $\mathrm{mm}$ at 15 angular positions (24 degrees apart). Linearity and uniformity corrections were applied based on calibration data acquired from the selected top DOI layers. The final ML-EM image reconstruction (20 iterations) was carried out following DOI correction in each layer (4-layer). The reconstruction was repeated with data identified in the top two layers, eliminating events that interacted deeper in the detector (2-layer). The results are compared with reconstruction of all data without DOI (Fig. 13).

The resulting reconstructed images illustrate improved resolution compared to the non-DOI case, particularly noticeable in the proximity of the detector gap. This is consistent with the simulated results. The measured short and long axis resolution is reported (Fig. 14). Results illustrate the improved resolution when the deeper layers are removed, especially for the long axis. The reduced sampling in the detector gap region causes a large deviation in the measurement, particularly at the edge of the FOV $(100 \mathrm{~mm})$. The error in the deep layer positioning resulted in little improvement in the 4-layer DOI correction. Using the 2 top layers only presented consistent improvement in resolution ranging from $6.26 \%$ to $16.88 \%$ for long-axis resolution and $5.86 \%$ to $8.96 \%$ for short-axis resolution. A paired t-test of the long and short axis resolution demonstrated significant improvement for 2 layer DOI-corrected data, independent of radius $(\mathrm{p}<0.001)$.

\section{DISCUSSION}

In this paper the objective was to identify DOI and to illustrate the effect of including DOI information specifically for the INSERT system. Improvement in image quality by reducing parallax effects was clearly demonstrated in simulation studies. Also the implementation of a 4 layer configuration was demonstrated to be effective on a single well-calibrated desk-top detector ( $30 \%$ improvement on a single axis).

However, implementation on the constructed prototype INSERT system exposed some limitations in the event positioning algorithm for deeper DOI layers close to the readout plane. There may be several reasons for this deficiency. To limit the number of readout channels the signals were summed over $4 \mathrm{SiPMs}$ and so the event discrimination close to the readout plane becomes very much peaked. Additionally, the system has aged with possible breakdown of optical coupling on some detectors. As a result the event positioning algorithm may require detector-specific refinement in order to rectify the observed problems.

Nevertheless the availability of DOI information was useful in identifying the issues and enabled the rejection of prob- 


\section{b)}

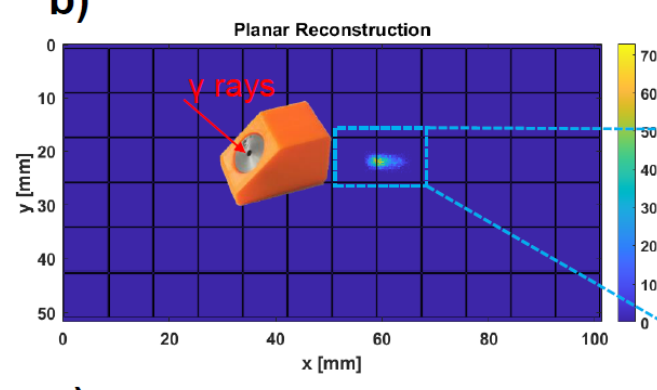

c)
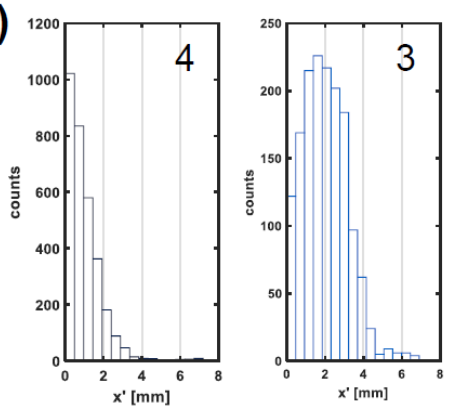
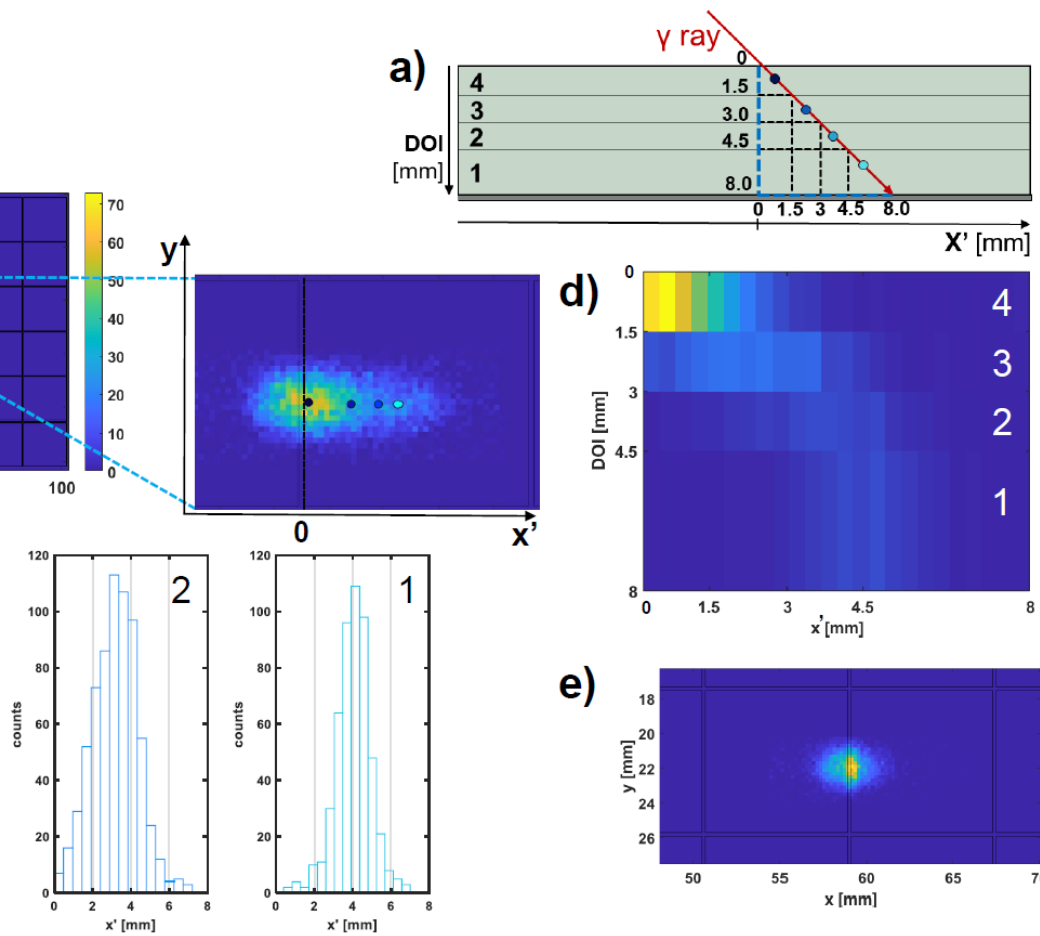

d)

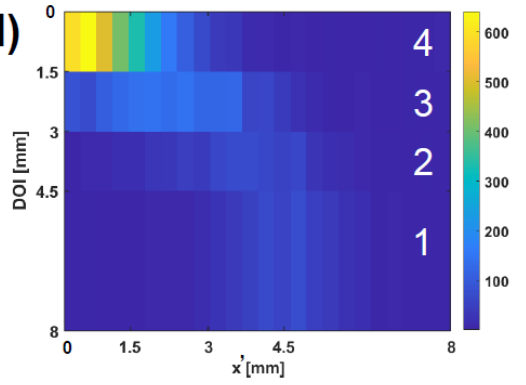

e)

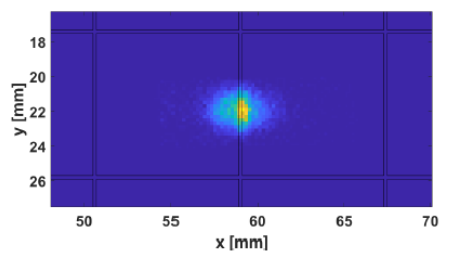

Fig. 11. a) Schematic view of the tilted irradiation (45 degrees). The DOI ranges of the four classes are directly mapped into the respective $x$ ' ranges. b) Planar reconstruction of the tilted irradiation. The gamma beam enters the crystal directed at $45^{\circ}$ towards the right, leading to an elongation on the right of the reconstructed spot. c) Distribution of the reconstructed $x$ ' for each DOI class. d) Reconstruction of the interaction positions on the $x$-DOI plane. e) Projected spot correction showing the recovering of the round shape.
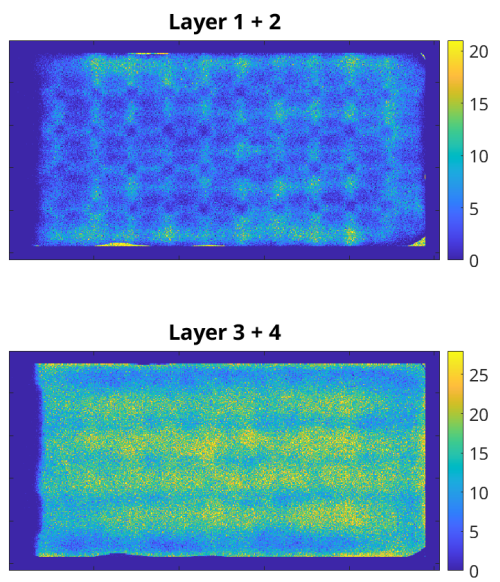

Fig. 12. A uniformity flood is separated into 4 DOI layers. The deepest layers present poor event localisation and can be eliminated with the DOI estimation algorithm. The top two layers are retained and used to calibrate the detectors.

lematic data to improve overall performance, increasing in the extrinsic spatial resolution of about $10 \%$.

This analysis has been performed for photons of energy in the $122-140 \mathrm{keV}$ range. For higher energies, DOI correction becomes more important, since there is greater likelihood of a deeper interaction. It may be preferable to choose in this case a different set of DOI layers, that best suits the interaction profile. A thicker crystal might be needed and energy-specific calibration of the LUTs is required.
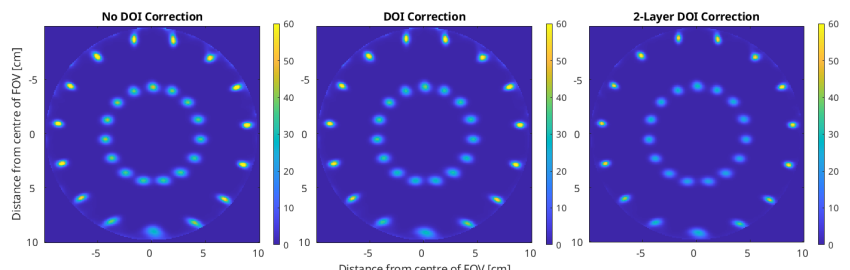

Fig. 13. Capillaries at 50 and $100 \mathrm{~mm}$ radius shown. The uncorrected image (left) demonstrates the degradation at the edge of the FOV and particularly in the gap region. The 4-layer DOI correction (centre) was limited by the deep layer calibration. Removing the deepest layers presents an improved DOI correction (right), however, reduces the reconstructed count from 138,900 to 93,077 .

\section{CONCLUSION}

We have presented a method to estimate the DOI in 8$\mathrm{mm}$ thick monolithic scintillator slabs coupled to arrays of SiPMs. This statistical method sorts events into 4 layers, whose thickness has been optimized by means of simulations and it leverages a ring pattern of SiPM signals. The main advantage of the method is the simple training step, requiring only flood field irradiations, already carried out routinely to perform other calibrations such as uniformity. No special collimators or training setups are required, making this solution appealing for SPECT scanners composed of several detectors (20 in our case) and to be operated in challenging clinical environment, such as multi-modal SPECT/MRI. The method has been experimentally validated and the improvement in spatial resolution has been demonstrated by both simulations and experimental 

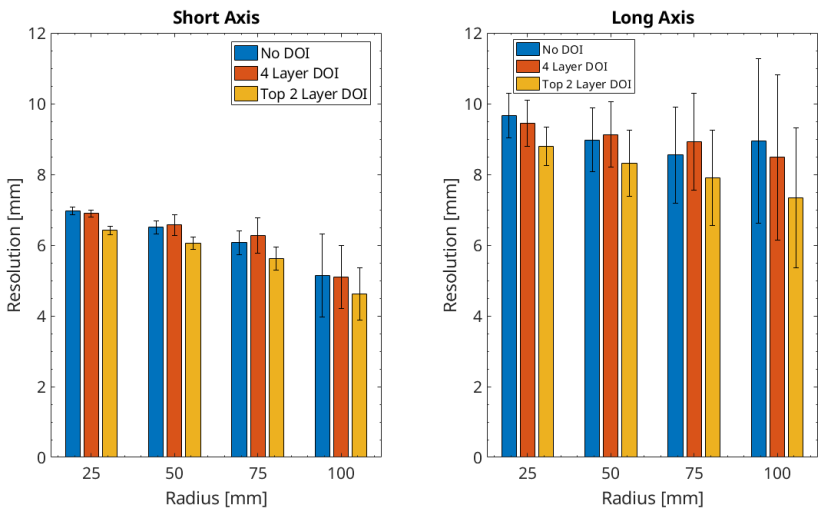

Fig. 14. Measurements of FWHM at 4 radii from the centre of the FOV. Resolution is measured across short and long axes of the capillary. The 2layer DOI correction presents the greatest improvement as the calibration is not limited by the poor event positioning in deeper layers.

results, for both a single detector (planar reconstruction of a tilted beam) and the full scanner (tomographic reconstruction).

\section{ACKNOWLEDGMENTS}

All authors declare that they have no known conflicts of interest in terms of competing financial interests or personal relationships that could have an influence or are relevant to the work reported in this paper. The Institute of Nuclear Medicine receives support from the NIHR UCLH Biomedical Research Centre. AM is funded through the UCL EPSRC Centre for Doctoral Training in Intelligent Integrated Imaging in Healthcare.

\section{REFERENCES}

[1] B. F. Hutton, M. Occhipinti, A. Kuehne, D. Máthé, N. Kovács, H. Waiczies, K. Erlandsson, D. Salvado, M. Carminati, G. L. Montagnani, S. C. Short, L. Ottobrini, P. van Mullekom, C. Piemonte, T. Bukki, Z. Nyitrai, Z. Papp, K. Nagy, T. Niendorf, I. de Francesco, and C. a. Fiorini, "Development of clinical simultaneous spect/mri," The British Journal of Radiology, vol. 91, no. 1081, p. 20160690, 2018, pMID: 28008775. [Online]. Available: https://doi.org/10.1259/bjr.20160690

[2] P. Busca, C. Fiorini, A. Butt, M. Occhipinti, R. Peloso, R. Quaglia, F. Schembari, P. Trigilio, G. Nemeth, P. Major, K. Erlandsson, and B. Hutton, "Simulation of the expected performance of insert: A new multi-modality spect/mri system for preclinical and clinical imaging," Nuclear Instruments and Methods in Physics Research Section A: Accelerators, Spectrometers, Detectors and Associated Equipment, vol. 734, pp. 141-146, 2014, pSMR2013 - PET-MR and SPECT-MR: Current status of Instrumentation, Applications and Developments. [Online]. Available: https://www.sciencedirect.com/science/article/pii/S0168900213011972

[3] M. Carminati, I. D'Adda, A. J. Morahan, K. Erlandsson, K. Nagy, M. Czeller, B. Tölgyesi, Z. Nyitrai, A. Savi, P. van Mullekom, B. F. Hutton, and C. Fiorini, "Clinical sipm-based mri-compatible spect: Preliminary characterization," IEEE Transactions on Radiation and Plasma Medical Sciences, vol. 4, no. 3, pp. 371-377, 2020.

[4] A. González-Montoro, A. Aguilar, G. Cañizares, P. Conde, L. Hernández, L. F. Vidal, M. Galasso, A. Fabbri, F. Sánchez, J. M. Benlloch et al., "Performance study of a large monolithic lyso pet detector with accurate photon doi using retroreflector layers," IEEE Transactions on Radiation and Plasma Medical Sciences, vol. 1, no. 3, pp. 229-237, 2017.

[5] H. T. Van Dam, S. Seifert, R. Vinke, P. Dendooven, H. Löhner, F. J. Beekman, and D. R. Schaart, "A practical method for depth of interaction determination in monolithic scintillator pet detectors," Physics in Medicine \& Biology, vol. 56, no. 13, p. 4135, 2011.
[6] G. Borghi, V. Tabacchini, R. Bakker, and D. R. Schaart, "Sub-3 mm, near-200 ps tof/doi-pet imaging with monolithic scintillator detectors in a $70 \mathrm{~cm}$ diameter tomographic setup," Physics in Medicine \& Biology, vol. 63 , no. 15 , p. $155006,2018$.

[7] Y. Wang, W. Zhu, X. Cheng, and D. Li, "3d position estimation using an artificial neural network for a continuous scintillator pet detector," Physics in Medicine \& Biology, vol. 58, no. 5, p. 1375, 2013.

[8] S. Pedemonte, L. Pierce, and K. Van Leemput, "A machine learning method for fast and accurate characterization of depth-of-interaction gamma cameras," Physics in Medicine \& Biology, vol. 62, no. 21, p. 8376, 2017.

[9] F. Müller, D. Schug, P. Hallen, J. Grahe, and V. Schulz, "A novel doi positioning algorithm for monolithic scintillator crystals in pet based on gradient tree boosting," IEEE Transactions on Radiation and Plasma Medical Sciences, vol. 3, no. 4, pp. 465-474, 2018.

[10] L. Bläckberg, S. Sajedi, O. A. Anderson, Y. Feng, G. El Fakhri, L. Furenlid, and H. Sabet, "Dynamic cardiac spect for diagnostic and theranostics applications: latest results," in 2020 IEEE Nuclear Science Symposium and Medical Imaging Conference (NSS/MIC). IEEE, pp. $1-3$.

[11] M. A. Korevaar, J. W. Heemskerk, and F. J. Beekman, "A pinhole gamma camera with optical depth-of-interaction elimination," Physics in Medicine \& Biology, vol. 54, no. 13, p. N267, 2009.

[12] M. Bettiol, E. Preziosi, M. Cinti, C. Borrazzo, A. Fabbri, B. Cassano, C. Polito, R. Pellegrini, and R. Pani, "A depth-of-interaction encoding method for spect monolithic scintillation detectors," Journal of Instrumentation, vol. 11, no. 12, p. C12054, 2016.

[13] M. Ljungberg and S.-E. Strand, "A monte carlo program for the simulation of scintillation camera characteristics," Computer Methods and Programs in Biomedicine, vol. 29 no. 4, pp. 257 - 272, 1989. [Online]. Available: http://www.sciencedirect.com/science/article/pii/0169260789901119

[14] F. Neves, V. Solovov, V. Chepel, M. Lopes, J. P. da Cunha, A. Lindote, and C. Silva, "Position reconstruction in a liquid xenon scintillation chamber for low-energy nuclear recoils and $\gamma$-rays," Nuclear Instruments and Methods in Physics Research Section A: Accelerators, Spectrometers, Detectors and Associated Equipment, vol. 573, no. 1-2, pp. 48-52, 2007.

[15] R. S. Miyaoka, X. Li, W. Hunter, L. A. Pierce, W. McDougald, P. E. Kinahan, and T. K. Lewellen, "Resolution properties of a prototype continuous miniature crystal element (cmice) scanner," IEEE transactions on nuclear science, vol. 58, no. 5, pp. 2244-2249, 2011.

[16] M. Occhipinti, M. Carminati, P. Busca, A. Butt, G. Montagnani, P. Trigilio, C. Piemonte, A. Ferri, A. Gola, T. Bukki et al., "Characterization of the detection module of the insert spect/mri clinical system," IEEE Transactions on Radiation and Plasma Medical Sciences, vol. 2, no. 6, pp. 554-563, 2018.

[17] A. Morozov, F. Alves, J. Marcos, R. Martins, L. Pereira, V. Solovov, and V. Chepel, "Iterative reconstruction of sipm light response functions in a square-shaped compact gamma camera," Physics in Medicine \& Biology, vol. 62, no. 9, p. 3619, 2017.

[18] V. Solovov, V. Belov, D. Y. Akimov, H. Araujo, E. Barnes, A. Burenkov, V. Chepel, A. Currie, L. DeViveiros, B. Edwards et al., "Position reconstruction in a dual phase xenon scintillation detector," IEEE Transactions on Nuclear Science, vol. 59, no. 6, pp. 3286-3293, 2012.

[19] A. Morozov, V. Solovov, R. Martins, F. Neves, V. Domingos, and V. Chepel, "Ants2 package: simulation and experimental data processing for anger camera type detectors," Journal of Instrumentation, vol. 11, no. 04, p. P04022, 2016.

[20] T. Ling, T. Lewellen, and R. Miyaoka, "Depth of interaction decoding of a continuous crystal detector module," Physics in Medicine \& Biology, vol. 52, no. 8, p. 2213, 2007.

[21] H. T. Van Dam, S. Seifert, R. Vinke, P. Dendooven, H. Löhner, F. J. Beekman, and D. R. Schaart, "A practical method for depth of interaction determination in monolithic scintillator pet detectors," Physics in Medicine \& Biology, vol. 56, no. 13, p. 4135, 2011.

[22] R. Pani, S. Nourbakhsh, P. Pani, P. Bennati, S. L. Meo, M. N. Cinti, R. Pellegrini, B. Cassano, M. Bettiol, and R. Scafe, "Continuous doi determination by gaussian modelling of linear and non-linear scintillation light distributions," in 2011 IEEE Nuclear Science Symposium Conference Record. IEEE, 2011, pp. 3386-3389. 